\title{
Wall-Driven Versus Pressure Gradient-Driven Flows in Porous Media
}

\author{
Eugen Magyari
}

Received: 18 December 2013 / Accepted: 10 February 2014 / Published online: 23 February 2014

(C) Springer Science+Business Media Dordrecht 2014

\begin{abstract}
The heat transfer characteristics of two boundary layer flows past an isothermal plane surface adjacent to a saturated Darcy-Brinkman porous medium is compared to each other in this paper. The flows are driven either by a stretching of the adjacent plane boundary, or by an external pressure gradient. It is found that below a threshold value $\tilde{P} r_{*}$ of the modified Prandtl number $\tilde{P} r$, the Nussselt number in case of the pressure gradient-driven flow is larger than in case of the wall- driven flow, while for $\tilde{P} r>\tilde{P} r_{*}$ the flow driven by the moving wall provides a more efficient heat transfer mechanism. The dependence of $\tilde{P} r_{*}$ on the Darcy number is also discussed in detail.
\end{abstract}

Keywords Darcy-Brinkman porous medium · Wall-driven flows · Pressure gradient-driven flows $\cdot$ Heat transfer $\cdot$ Nusselt number $\cdot$ Modified Prandtl number

\section{Introduction}

The motivation of the present paper originates from a remark of Nield and Bejan read in the fourth edition of their prestigious book (Nield and Bejan 2013, p.174) which asserts that "Despite the fact that a stretching sheet has a negligible effect on flow in a practical porous medium, papers on this topic have been published by..." (there are listed 25 papers, but to our rating the number of papers published in this research field is much larger).

Physical intuition suggests indeed that due to the flow resistance of the solid matrix, the effect of a sliding boundary adjacent to a saturated porous medium gets attenuated inside the medium rapidly, so that the viscous stress transferred from the moving boundary to the saturating fluid becomes perceptive in a thin neighboring layer only. Nevertheless, the boundary layer theory tells us that important flow and heat transfer phenomena may have their origin just in this thin velocity boundary layer induced by the moving boundary. In

\section{E. Magyari ( $\square)$}

Departement Physik, Theoretische Physik, Universität Basel, Klingelbergstrasse

82, 4056 Basel, Switzerland

e-mail: magyari@bluewin.ch 
order to clarify this question, the present paper undertakes a quantitative comparison of the heat transfer characteristics of the boundary layer flows past an isothermal plane surface adjacent to a Darcy-Brinkman porous medium, assuming that the fluid motion is driven either by a stretching of the plane boundary of the medium, or by an applied pressure gradient. Surprisingly it is found that above a threshold value $\tilde{P} r_{*}$ of the modified Prandtl number (which depends on the Darcy number) the wall-driven flows provide a more efficient heat transfer mechanism (even when the wall motion exerts a negligible effect on the flow in the bulk of the porous medium).

\section{Model and Problem Formulation}

We consider a semi-infinite flat plate of constant temperature $T_{\mathrm{w}}$ adjacent to a DarcyBrinkman porous medium saturated with an incompressible viscous fluid of ambient temperature $T_{\infty}<T_{\mathrm{w}}$. Over the plate a boundary layer flow is driven simultaneously by (i) a non-uniform motion of the plate which is stretched in the $x$-direction with a linearly rising velocity $u_{\mathrm{w}}=a x$, as well as by (ii) a pressure gradient $-\partial p / \partial x$ corresponding to the asymptotic velocity distribution $u_{\infty}=b x$ at large transverse distances $y$ from the plate. The governing continuity, Darcy-Brinkman and energy equations of this "doubly driven flow" are

$$
\begin{aligned}
\frac{\partial u}{\partial x}+\frac{\partial v}{\partial y} & =0, \\
\frac{\rho_{f}}{\varphi^{2}}\left(u \frac{\partial u}{\partial x}+v \frac{\partial u}{\partial y}\right) & =-\frac{\partial p}{\partial x}+\mu_{e f f} \frac{\partial^{2} u}{\partial y^{2}}-\frac{\mu}{K} u, \\
u \frac{\partial T}{\partial x}+v \frac{\partial T}{\partial y} & =\alpha_{m} \frac{\partial^{2} T}{\partial y^{2}}
\end{aligned}
$$

being subject to the boundary conditions

$$
\begin{aligned}
& u \equiv u_{\mathrm{W}}=a x, v=0, T \equiv T_{\mathrm{W}}=\text { const. at } y=0, \\
& u \rightarrow u_{\infty}=b x, T \rightarrow T_{\infty}=\text { const. } \quad \text { as } y \rightarrow \infty .
\end{aligned}
$$

In the above equations $\rho_{f}$ is the fluid density, $\mu_{\text {eff }}$ the effective viscosity, $\varphi$ the porosity, $K$ the permeability of the medium, and the other notation is standard. For further details concerning the Darcy-Brinkman Eq. (2) see Nield 2007; Kumaran and Tamizharasi 2011 and Nield and Bejan (2013), pp. 15-17.

The first asymptotic condition (5) and Eq. (2) imply

$$
-\frac{1}{\rho_{f}} \frac{\partial p}{\partial x}=\frac{1}{\varphi^{2}} u_{\infty} \frac{d u_{\infty}}{d x}+\frac{v}{K} u_{\infty}
$$

so that Eq. (2) becomes

$$
u \frac{\partial u}{\partial x}+v \frac{\partial u}{\partial y}=v_{e f f} \varphi^{2} \frac{\partial^{2} u}{\partial y^{2}}-\frac{v \varphi^{2}}{K}(u-b x)+b^{2} x,
$$

where $v=\mu / \rho_{f}$ and $v_{e f f}=\mu_{e f f} / \rho_{f}$.

The two driving forces of the flow, due, respectively, to the wall motion and the external pressure gradient, can support each other resulting in an aiding flow $(a, b>0)$, or can be adverse to each other, leading to an opposing flow $(a<0, b>0)$. In the latter case, one 
usually speaks about a shrinking surface. For the comparative approach of the present paper, however, mainly the two limiting cases of these doubly driven flows will be of interest, namely (i) the case in which the flow is driven solely by the stretching wall $(a>0, b=0)$ and (ii) the case in which the flow is driven solely by the external pressure gradient $(a=0, b>0)$. The aiding case $a=b$ of the doubly driven flow which admits a simple analytical solution will also be addressed shortly.

\section{Similarity Solutions}

With the aid of the similarity transformations

$$
\begin{aligned}
& \psi=\varphi \sqrt{(a+b) v_{e f f}} x f(\eta), \eta=\frac{1}{\varphi} \sqrt{\frac{a+b}{v_{e f f}} y} \\
& T=T_{\infty}+\Delta T \theta(\eta), \Delta T=T_{w}-T_{\infty}
\end{aligned}
$$

where the stream function is related to the velocity components $(u, v)$ by the usual definition $u=\partial \psi / \partial y, v=-\partial \psi / \partial x$, the boundary value problem specified by Eqs. (1-6) reduces to

$$
\begin{aligned}
& f^{\prime \prime \prime}+f f^{\prime \prime}+\varepsilon^{2}-f^{\prime 2}+\lambda\left(\varepsilon-f^{\prime}\right)=0, \\
& \theta^{\prime \prime}+\tilde{P} r f \theta^{\prime}=0, \\
& f(0)=0, f^{\prime}(0)=1-\varepsilon, f^{\prime}(\infty)=\varepsilon, \\
& \theta(0)=1, \theta(\infty)=0 .
\end{aligned}
$$

Here, the primes denote differentiations with respect to $\eta$, and

$$
\varepsilon=\frac{b}{a+b}, \lambda=\frac{v \varphi^{2}}{(a+b) K}, P \tilde{r}=\varphi^{2} \frac{v_{e f f}}{\alpha_{m}} .
$$

The symbol $\tilde{P} r$ has the meaning of a modified Prandtl number, $\lambda$ the meaning of a modified reciprocal Darcy number $1 / \tilde{D} a=L^{2} / K$ with the characteristic length $L=\varphi \sqrt{v /(a+b)}$, and plays in our consideration the role of a dichotomic variable taking the values 0 and 1 , allowing for a simple switching between the wall-driven flows $(b=0, \varepsilon=0)$ and the pressure gradient-driven flows $(a=0, \varepsilon=1)$, respectively. The velocity components are obtained in terms of the solution of the flow boundary value problem (9), (11) by

$$
u=\left(u_{w}+u_{\infty}\right) f^{\prime}(\eta), v=-\varphi \sqrt{(a+b) v_{e f f}} f(\eta) .
$$

\section{The Wall-Driven Flows $(\varepsilon=0, a>0)$}

In case $\varepsilon=0$ the flow problem (9), (11) reduces to

$$
\begin{aligned}
& f^{\prime \prime \prime}+f f^{\prime \prime}-f^{\prime 2}-\lambda f^{\prime}=0, \\
& f(0)=0, f^{\prime}(0)=1, f^{\prime}(\infty)=0
\end{aligned}
$$

and admits the exact solution

$$
f(\eta)=\frac{1-e^{-\sqrt{1+\lambda} \eta}}{\sqrt{1+\lambda}}, \quad \lambda=\frac{v \varphi^{2}}{a K}, \eta=\frac{1}{\varphi} \sqrt{\frac{a}{v_{e f f}}} y .
$$


Thus, the velocity boundary layer $f^{\prime}(\eta)=e^{-\sqrt{1+\lambda} \eta} \equiv e^{-y / \delta}$ possesses in this case the (dimensional) thickness

$$
\delta=\sqrt{\frac{v_{e f f}}{v}\left(1+\frac{a K}{v \varphi^{2}}\right)^{-1} K} .
$$

The boundary value problem (15) and its exact solution (16), as well as the corresponding temperature problem (10), (12) can be encountered in various contexts of the fluid mechanics. In this respect, a short historical survey is outlined in Appendix 1.

The solution of the associated temperature problem (10), (12) can simply be obtained by two successive integrations of Eq. (10) and the result can be put in the form

$$
\theta(\eta)=1+\theta^{\prime}(0) \frac{\exp \left(\frac{\tilde{P} r}{1+\lambda}\right)}{\sqrt{1+\lambda}} \int_{0}^{\sqrt{1+\lambda} \eta} \exp \left[-\frac{\tilde{P} r}{1+\lambda}\left(z+e^{-z}\right)\right] d z .
$$

Letting here $\eta \rightarrow \infty$ and taking into account the asymptotic condition $\theta(\infty)=0$ one immediately obtains for the reduced Nusselt number $N u=-\theta^{\prime}(0)$ the expression

$$
N u=\frac{\sqrt{1+\lambda} \exp \left(-\frac{\tilde{P} r}{1+\lambda}\right)}{\int_{0}^{\infty} \exp \left[-\frac{\tilde{P} r}{1+\lambda}\left(z+e^{-z}\right)\right] d z} .
$$

The exact solution (18) can alternatively be expressed also in terms of Kummer's confluent hypergeometric function $M(a, b, z)$ as (see Abramowitz and Stegun 1972, Chapter 13)

$$
\theta(\eta)=e^{-\frac{\tilde{P} r}{\sqrt{1+\lambda}}[\eta-f(\eta)]} \frac{M\left(1,1+\frac{\tilde{P} r}{1+\lambda}, \frac{\tilde{P} r}{1+\lambda} e^{-\sqrt{1+\lambda} \eta}\right)}{M\left(1,1+\frac{\tilde{P} r}{1+\lambda}, \frac{\tilde{P} r}{1+\lambda}\right)},
$$

where $f(\eta)$ is given by Eq. (16). Accordingly, the Nusselt number (19) changes to the equivalent form

$$
N u=\frac{\sqrt{1+\lambda} \tilde{P} r}{1+\lambda+\tilde{P} r} \frac{M\left(2,2+\frac{\tilde{P} r}{1+\lambda}, \frac{\tilde{P} r}{1+\lambda}\right)}{M\left(1,1+\frac{\tilde{P} r}{1+\lambda}, \frac{\tilde{P} r}{1+\lambda}\right)} .
$$

The red curve of Fig. 1 illustrates the dependence of the Nusselt number given by Eq. (19) or Eq. (21) on the modified Prandtl number $\tilde{P} r$ for the selected value $\lambda=1$ of the reciprocal Darcy number.

\section{The Pressure Gradient-Driven Flows $(\varepsilon=1, b>0)$}

In case $\varepsilon=1$ the flow problem (9), (11) reduces to

$$
\begin{aligned}
& f^{\prime \prime \prime}+f f^{\prime \prime}+1-f^{\prime 2}+\lambda\left(1-f^{\prime}\right)=0, \\
& f(0)=0, f^{\prime}(0)=0, f^{\prime}(\infty)=1
\end{aligned}
$$

where now $\lambda=v \varphi^{2} /(b K)$.

Unfortunately, no exact analytical solution of this problem is known. Its numerical solution, however, is a standard task. Once this solution has been found, the solution of the 


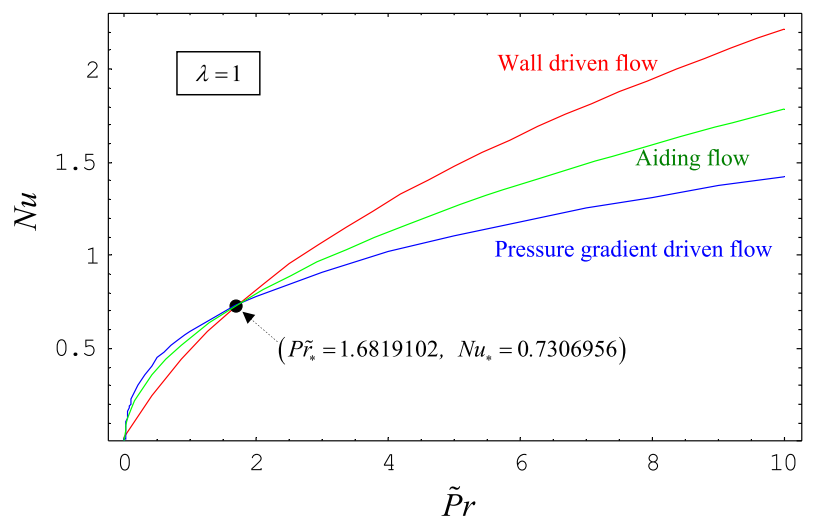

Fig. 1 Dependence of the reduced Nusselt number $N u=-\theta^{\prime}(0)$ on the modified Prandtl number $\tilde{P} r$ in case of wall-driven flow (red curve, $\varepsilon=0$ ) and the pressure gradient-driven flow (blue curve, $\varepsilon=1$ ) for the value $\lambda=1$ of the reciprocal Darcy number. The green curve corresponds to the doubly driven aiding flow with $a=b>0,(\varepsilon=1 / 2)$. The dot marks the intersection point of the red and blue curves at $\left(\tilde{P} r_{*}=1.6819102, N u_{*}=0.7306956\right)$. At the same $\tilde{P} r=\tilde{P} r_{*}$, the Nusselt number corresponding to the aiding flow is quite close to $N u_{*}$ having the value $N u=\sqrt{\tilde{P} r_{*} / \pi}=0.7317$

corresponding temperature problem (10), (12) can be obtained, similar to the case of walldriven flows, by two successive integrations of Eq. (10), resulting in

$$
\theta(\eta)=1-N u \int_{0}^{\eta} \exp \left[-\tilde{P} r \int_{0}^{\eta_{2}} f\left(\eta_{1}\right) d \eta_{1}\right] d \eta_{2} .
$$

The corresponding reduced Nusselt number $N u=-\theta^{\prime}(0)$ is

$$
N u=\left(\int_{0}^{\infty} \exp \left[-\tilde{P} r \int_{0}^{\eta_{2}} f\left(\eta_{1}\right) d \eta_{1}\right] d \eta_{2}\right)^{-1} .
$$

For the selected value $\lambda=1$ of the reciprocal Darcy number the numerical solution of the flow problem (22) is obtained with $f^{\prime \prime}(0)=1.585333$ and the dependence of the corresponding Nusselt number (24) on the modified Prandtl number $\tilde{P} r$ is represented by the blue curve of Fig. 1.

The red and blue curves of Fig. 1 illustrate the main result of the present paper, showing that for any specified value of the reciprocal Darcy number $\lambda$, a threshold value $\tilde{P} r_{*}$ of the modified Prandtl number $\tilde{P} r$ exists so that below $\tilde{P} r_{*}$ the pressure gradient-driven flow and above of $\tilde{P} r_{*}$ the wall-driven flow provides a more efficient heat transfer mechanism, respectively. It is worth mentioning here that for the recovery factors (the reciprocal Nusselt numbers) of Pohlhausen's classical plate thermometer in an external stream, and the moving plate thermometer in a quiescent viscous fluid, a similar dependence on the Prandtl number has been found (see Magyari 2008).

The green curve of Fig. 1 between the red and blue ones corresponds to the special case $a=b>0$ of the "doubly driven" aiding flows which will be considered in the next section shortly. 
Table 1 Scaling Behaviour of the reduced Nusselt number $N u=-\theta^{\prime}(0)$ for small and large values of the modified Prandtl number $\tilde{P} r$ in the three indicated cases

\begin{tabular}{llll}
\hline$\tilde{P} r$ & $\begin{array}{l}\text { Wall-driven flow } \\
(\varepsilon=0, \lambda=1)\end{array}$ & $\begin{array}{l}\text { Pressure gradient-driven } \\
\text { flow }(\varepsilon=1, \lambda=1)\end{array}$ & $\begin{array}{l}\text { Doubly driven aiding } \\
\text { flow }(\varepsilon=1 / 2)\end{array}$ \\
\hline$\tilde{P} r<<1$ & $N u=0.70 \cdot \tilde{P} r$ & $N u=0.79 \cdot \tilde{P} r^{1 / 2}$ & $N u=(\tilde{P} r / \pi)^{1 / 2}=0.5642 \cdot \tilde{P} r^{1 / 2}$ \\
$\tilde{P} r>>1$ & $N u=0.78 \cdot \tilde{P} r^{1 / 2}$ & $N u=0.71 \cdot \tilde{P} r^{1 / 3}$ & \\
\hline
\end{tabular}

\section{The Aiding Flow Case $a=b(\varepsilon=1 / 2)$}

In the special case $a=b(\varepsilon=1 / 2)$ of the aiding flows $(a, b>0,0<\varepsilon<1)$ which is driven simultaneously by a pressure gradient and the wall motion, the flow problem (9), (11) admits the exact elementary solution $f(\eta)=\eta / 2$ which is independent of $\lambda$. Thus, the temperature problem (10), (12) can also be solved exactly. This solution, as well as the corresponding reduced Nusselt number $N u=-\theta^{\prime}(0)$, is

$$
\theta(\eta)=\operatorname{erfc}\left(\frac{\sqrt{\tilde{P} r}}{2} \eta\right)
$$

and

$$
N u=\sqrt{\frac{\tilde{P} r}{\pi}}=0.5642 \cdot \tilde{P} r^{1 / 2}
$$

respectively.

As expected, the green curve of Fig. 1 corresponding to Eq. (26) is located between the red and blue curves seen in the same Figure. The dot, however, does not represent a triple intersection point. It is only the intersection point of the red and blue curves at $\left(\tilde{P} r_{*}, N u_{*}\right) \cong$ $(1.6819,0.7307)$, while the Nusselt number (26) corresponding to the aiding flow has at $\tilde{P} r=\tilde{P} r_{*}$ the value $N u=0.7317$ which, however, is quite close to $N u_{*}=0.7307$. It is also worth emphasizing again that the shape of the green curve is independent on $\lambda$. The case of the triple intersection point will be addressed in Sect. 8.

\section{Scaling Behaviour of $N u$ for Small and Large Values of $\tilde{\boldsymbol{P}} \boldsymbol{r}$}

In Table 1 the correlation formulas of the reduced Nusselt number $N u$ and the modified Prandtl number $\tilde{P} r$ have been collected for the three boundary layer flows discussed in Sect. 4, 5 and 6 , respectively. These correlations are in a full agreement with the shape of the three curves plotted in Fig. 1.

\section{Dependence of $\tilde{\boldsymbol{P}} \boldsymbol{r}_{*}$ on $\lambda$}

The numerical results reported in Sect. 4-7 correspond to the selected value $\lambda=1$ of the reciprocal Darcy number. The aim of the present Section is to relax this restriction and to examine the dependence of the threshold value $\tilde{P} r_{*}$ on $\lambda$. The following two examples corresponding, respectively, to $\lambda=0$ and $\lambda=10$ show that this dependence is sensitive. In 


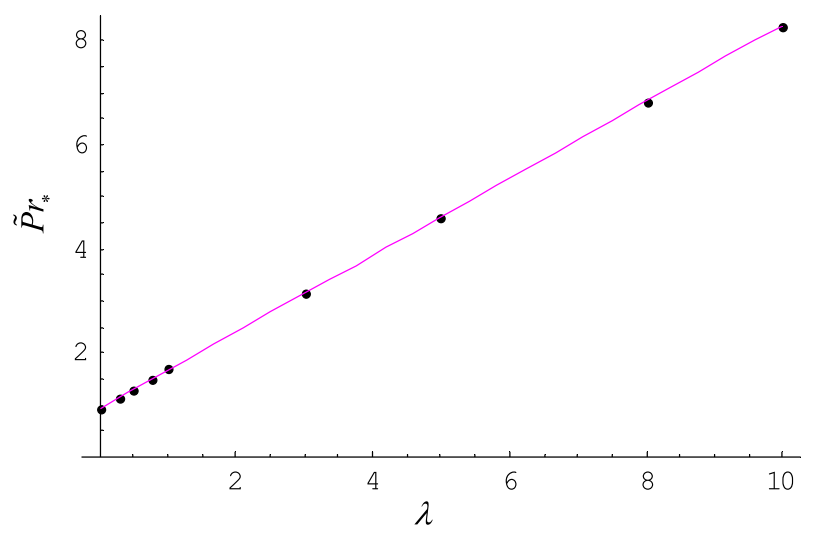

Fig. 2 Dependence of the threshold value $\tilde{P} r_{*}$ of the modified Prandtl number $\tilde{P} r$ on the modified reciprocal Darcy number. The selected values $\lambda=0,0.3,0.5,0.776528,1,3,5,8$ and $\lambda=10$ are marked by dots on the straight line corresponding to Eq. (27)

case $\lambda=0$ the boundary value problem (22) of the pressure gradient-driven flows coincide formally with the classical problem of the plane Hiemenz flow, and thus $f^{\prime \prime}(0)=1.232589$. For the threshold value $\tilde{P} r_{*}$ and the corresponding Nusselt number one finds in this case $\left(\tilde{P} r_{*}, N u_{*}\right)_{\lambda=0}=(0.933818,0.555437)$. In case $\lambda=10$, on the other hand, one finds $f^{\prime \prime}(0)=3.391674$ and $\left(\tilde{P} r_{*}, N u_{*}\right)_{\lambda=10}=(8.281379,1.585547)$. In Fig. 2 the dependence of $\tilde{P} r_{*}$ on $\lambda$ has been plotted for $\lambda=0,0.3,0.5,0.776528,1,3,5,8$ and $\lambda=10$. The straight line

$$
\tilde{P} r_{*}=0.734756 \cdot \lambda+0.933818
$$

which connects the points corresponding to $\lambda=0$ and $\lambda=10$ yields an acceptable approximation also for the intermediate points (marked in Fig. 2 by dots). It is worth mentioning here that the approximation (27) performs well also in the range $\lambda>10$. For example, extrapolated to $\lambda=100$, Eq. (27) yields $\tilde{P} r_{*}=74.409418$. The solution of the corresponding flow problem (22) is obtained with $f^{\prime \prime}(0)=10.074741$. The Nusselt number at the threshold value $\tilde{P} r_{*}=74.409418$ can then be calculated either from Eq. (21) or (24). The result is $N u_{*}=4.731239$.

In Table 2 the "exact numerical" values of $\tilde{P} r_{*}$ and $N u_{*}$ corresponding to the dots seen in Fig. 2 have been collected. For convenience, in the last column of Table 2 also the values of the skin friction $f^{\prime \prime}(0)$ in case of the pressure gradient-driven flow have been included for the specified values of $\lambda$. A simple inspection Fig. 2 and of Table 2 shows that the threshold value $\tilde{P} r_{*}$ of the Prandtl number which marks the crossover from the heat transfer regime dominated by the pressure gradient flow $\left(\tilde{P} r<\tilde{P} r_{*}\right)$ to the regime dominated by the wall-driven flow $\left(\tilde{P} r>\tilde{P} r_{*}\right)$ increases with the increasing value of the reciprocal Darcy number $\lambda$. This behaviour is described approximately by the linear relationship (27). The corresponding Nussel number $N u_{*}$ is also an increasing function of $\lambda$. The special value $\lambda=0.776528$ of the reciprocal Darcy number corresponds to case in which the intersection point $\left(\tilde{P} r_{*}, N u_{*}\right)_{\lambda=0.776528}=(1.516147,0.695752)$ of the curves $N u=N u(\tilde{P} r)$ corresponding, respectively, to the wall-driven and pressure gradient-driven flow, is located exactly on the curve $N u=N u(\tilde{P} r)$ described by Eq. (26) of the aiding flow discussed in 
Table 2 Dependence of the threshold value $\tilde{P} r_{*}$ of the modified Prandtl number and of the corresponding Nusselt number $N u_{*}$ on the reciprocal Darcy number $\lambda$ (see also Fig. 2)

In the last column the values of the skin friction $f^{\prime \prime}(0)$ for the respective pressure gradient-driven flows have been included for convenience

\begin{tabular}{llll}
\hline$\lambda$ & $\tilde{P} r_{*}$ & $N u_{*}$ & $f^{\prime \prime}(0)$ \\
\hline 0 & 0.933818 & 0.555437 & 1.232589 \\
0.3 & 1.160474 & 0.613978 & 1.347956 \\
0.5 & 1.310224 & 0.649689 & 1.419765 \\
0.776528 & 1.516147 & 0.695752 & 1.513541 \\
1 & 1.681910 & 0.730696 & 1.585333 \\
3 & 3.154539 & 0.987958 & 2.123242 \\
5 & 4.620881 & 1.189951 & 2.550662 \\
8 & 6.817588 & 1.440454 & 3.082878 \\
10 & 8.281379 & 1.585547 & 3.391674
\end{tabular}

Sect. 6. In other words, in a counterpart of Fig. 1 prepared for $\lambda=0.776528$ (instead of $\lambda=1),\left(\tilde{P} r_{*}, N u_{*}\right)=(1.516147,0.695752)$ would represent a "triple point," where the Nusselt numbers of the three mentioned flows coincide exactly.

\section{Summary and Conclusions}

The heat transfer characteristics of the boundary layer flows driven in a saturated porous medium by a linear stretching of the adjacent plane boundary, or by an applied pressure gradient have been investigated in the paper. For every specified value of the reciprocal Darcy number $\lambda$ a threshold value $\tilde{P} r_{*}$ of the modified Prandtl number has been found which marks the crossover from the heat transfer regime dominated by the pressure gradient-driven flow $\left(\tilde{P} r<\tilde{P} r_{*}\right)$ to the regime dominated by the wall-driven flow $\left(\tilde{P} r>\tilde{P} r_{*}\right)$. Consequently, although the motion of an adjacent boundary exerts a weak effect on the flow in the bulk of a saturated porous medium, the heat transfer performance of such wall-driven flows may become dominant in comparison to the case of the "usual" pressure gradient-driven flows.

\section{Appendix 1: Historical Remark on the Exact Solution (16)}

The exact solution (16) of the boundary value problem (15) in fact is a slightly modified version of Crane's classical solution (Crane 1970)

$$
f(\eta)=1-e^{-\eta}
$$

of the same problem (15) for $\lambda=0$,

$$
\begin{aligned}
& f^{\prime \prime \prime}+f f^{\prime \prime}-f^{\prime 2}=0, \\
& f(0)=0, f^{\prime}(0)=1, f^{\prime}(\infty)=0 .
\end{aligned}
$$

Actually, Crane's solution (28) is an exact solution of the Navier-Stokes equation for the flow induced by a linearly stretching surface in a viscous fluid clear of solid material. It is the remarkable special case in which the boundary layer approximation becomes exact, since 
those terms of the Navier-Stokes equation which are usually neglected in the boundary layer theory, vanish in this case identically.

When the moving boundary is permeable and a lateral suction/injection of the fluid is applied, the impermeability condition $f(0)=0$ has to be replaced in (29) by $f(0)=f_{w}$ and, as a consequence, Crane's solution (28) is replaced by

$$
f(\eta)=f_{\mathrm{W}}+\frac{1}{\gamma}\left(1-e^{-\gamma \eta}\right), \gamma=\frac{f_{\mathrm{W}}}{2}+\sqrt{\left(\frac{f_{\mathrm{W}}}{2}\right)^{2}+1}
$$

The extension (30) of (28) has first been reported by Gupta and Gupta (1977).

Now, when in our present boundary value problem (15) with $\lambda \neq 0$ the impermeability condition $f(0)=0$ is replaced by $f(0)=f_{w}$, the solution (16) has to be replaced by

$$
f(\eta)=f_{\mathrm{W}}+\frac{1}{\gamma}\left(1-e^{-\gamma \eta}\right), \gamma=\frac{f_{\mathrm{W}}}{2}+\sqrt{\left(\frac{f_{\mathrm{W}}}{2}\right)^{2}+1+\lambda}
$$

Therefore, the linear term $-\lambda f^{\prime}$ of Eq. (15) adds to the extension (30) of Crane's basic solution (28) a simple algebraic modification only. This consists of the presence of $\lambda$ in the square root term of (31), of which sole effect is a change of the boundary layer thickness.

The boundary value problem (15) (with $f_{\mathrm{W}}=0$ or $f_{\mathrm{W}} \neq 0$ ) and its solution (31) is also encountered in case of the "hydromagnetic Crane flow." This flow is generated when the linearly stretching surface is submerged in an electrically conducting fluid (of electrical conductivity $\sigma$ ) and a uniform magnetic field $B_{0}$ is applied in the transverse direction. In this case the momentum equation has the form (see e.g. Chakrabarti and Gupta 1979)

$$
u \frac{\partial u}{\partial x}+v \frac{\partial u}{\partial y}=v \frac{\partial^{2} u}{\partial y^{2}}-\frac{\sigma B_{0}^{2}}{\rho} u
$$

It is immediately see that Eq. (32) is isomorphic to the case $b=0$ (wall-driven flows) of the Dracy-Brinkman equation (7)

$$
u \frac{\partial u}{\partial x}+v \frac{\partial u}{\partial y}=v_{e f f} \varphi^{2} \frac{\partial^{2} u}{\partial y^{2}}-\frac{v \varphi^{2}}{K} u
$$

when in Eq. (33) $v_{\text {eff }} \varphi^{2}$ is replaced by $v$ and $v \varphi^{2} / K$ by $\sigma B_{0}^{2} / \rho$. Thus, the hydromagnetic Crane problem based on the momentum equation (32) reduces to the present boundary value problem (15) with $\lambda$ replaced by $\tilde{\lambda}=\sigma B_{0}^{2} /(a \rho)$ and $\eta$ by $\tilde{\eta}=(a / v)^{1 / 2} y$. On this reason Eq. (16) (and Eq. (31) in case $f_{w} \neq 0$ ) represents at the same time also a solution of the hydromagnetic Crane problem. This isomorphism has recently been discussed by Pantokratoras (2009) in some detail.

Similarly to the way from Crane's flow solution (28) toward its extensions (30) and (31), a parallel development can be followed also with respect to the associated temperature solutions (with prescribed wall temperature distribution of power-law form). In this sense, the temperature solution associated with Crane's basic solution (28) has first been given in terms of the confluent hypergeometric function by Grubka and Bobba (1985). Then, the results of Grubka and Bobba (1985) have been extended to the case of the Gupta and Gupta solution (30) in the presence of suction or injection by Chen and Char (1988). In the mathematically equivalent context of the flow solution (31) of the present Darcy-Brinkman 
model, the temperature problem has been investigated numerically by Tamayol et al. (2010) and analytically by Fang and Zhang (2011). In this sense, similarly to the results of Chen and Char (1988), and of Fang and Zhang (2011), the form (20) of the present temperature solution represents a more or less straightforward extension of the pioneering results of Grubka and Bobba (1985).

\section{References}

Abramowitz, M., Stegun, I.A.: Handbook of Mathematical Functions. Dover Publications Inc., New York (1972)

Chakrabarti, A., Gupta, A.S.: Hydromagnetic flow and heat transfer over a stretching sheet. Quart. Appl. Math. 37, 73-78 (1979)

Chen, C.K., Char, M.I.: Heat transfer of a continuous stretching surface with suction or blowing. J. Math. Anal. Appl. 135, 568-580 (1988)

Crane, J.: Flow past a stretching plate. J. Appl. Math. Phys. (ZAMP) 21, 645-647 (1970)

Fang, T., Zhang, J.: Note on the heat transfer of flows over a stretching wall in porous media: exact solutions. Transp. Porous Med. 86, 579-584 (2011)

Grubka, L.J., Bobba, K.M.: Heat transfer characteristics of a continuous stretching surface with variable temperature. ASME J. Heat Transf. 107, 248-250 (1985)

Gupta, P.S., Gupta, A.S.: Heat and mass transfer on a stretching sheet with suction or blowing. Can. J. Chem. Eng. 55, 744-746 (1977)

Kumaran, V., Tamizharasi, R.: Brinkman flow past a stretching sheet. Transp. Porous Med. 87, 541-560 (2011)

Magyari, E.: The moving plate thermometer. Int. J. Therm. Sci. 47, 1436-1441 (2008)

Nield, D.A.: The modeling of viscous dissipation in a saturated porous medium. ASME J. Heat Transf. 129, 1459-1463 (2007)

Nield, D.A., Bejan, A.: Convection in Porous Media, 4th edn. Springer, New York (2013)

Pantokratoras, A.: Flow adjacent to a stretching permeable sheet in a Darcy-Brinkman porous medium. Transp. Porous Med. 80, 223-227 (2009)

Tamayol, A., Hooman, K., Bahrami, M.: Thermal analysis of flow in a porous medium over a permeable stretching wall. Transp. Porous Med. 85, 661-676 (2010) 